PROCEEDINGS OF THE

AMERICAN MATHEMATICAL SOCIETY

Volume 125, Number 10, October 1997, Pages 3033-3037

S 0002-9939(97)03915-4

\title{
THERE IS NO DEGREE INVARIANT HALF-JUMP
}

\author{
RODNEY G. DOWNEY AND RICHARD A. SHORE
}

(Communicated by Andreas R. Blass)

\begin{abstract}
We prove that there is no degree invariant solution to Post's problem that always gives an intermediate degree. In fact, assuming definable determinacy, if $W$ is any definable operator on degrees such that $\mathbf{a}<W(\mathbf{a})<\mathbf{a}^{\prime}$ on a cone then $W$ is low 2 or high 2 on a cone of degrees, i.e., there is a degree $\mathbf{c}$ such that $W(\mathbf{a})^{\prime \prime}=\mathbf{a}^{\prime \prime}$ for every $\mathbf{a} \geq \mathbf{c}$ or $W(\mathbf{a})^{\prime \prime}=\mathbf{a}^{\prime \prime \prime}$ for every $\mathbf{a} \geq \mathbf{c}$.
\end{abstract}

A striking phenomenon in the early days of computability theory was that every decision problem for axiomatizable theories turned out to be either decidable or of the same Turing degree as the halting problem $\left(\mathbf{0}^{\prime}\right.$, the complete computably enumerable set). Perhaps the most influential problem in computability theory over the past fifty years has been Post's problem [10] of finding an exception to this rule, i.e. a noncomputable incomplete computably enumerable degree. The problem has been solved many times in various settings and disguises but the solutions always involve specific constructions of strange sets, usually by the priority method that was first developed (Friedberg [2] and Muchnik [9]) to solve this problem. No natural decision problems or sets of any kind have been found that are neither computable nor complete. The question then becomes how to define what characterizes the natural computably enumerable degrees and show that none of them can supply a solution to Post's problem. Steel [13] suggests that a natural degree should be definable and its definition should relativize to an arbitrary degree (and so, in particular, be defined on degrees independently of the choice of representative). Along these lines an old question of Sacks' [11] asks whether there is a degree invariant solution to Post's problem, i.e. a computably enumerable degree invariant operator $W$ such that $A<_{T} W(A)<_{T} A^{\prime}$. (A function $W: 2^{\omega} \rightarrow 2^{\omega}$ is a computably enumerable operator if there is an $e \in \omega$ such that, for each set $A$, $W(A)=W_{e}^{A}$, the $e^{t h}$ set computably enumerable in $A$. Any function $f: 2^{\omega} \rightarrow 2^{\omega}$ is degree invariant if for every $A$ and $B, A \equiv_{T} B$ implies that $f(A) \equiv_{T} f(B)$.) Such an operator would clearly be a candidate for a natural solution to Post's problem.

Received by the editors February 16, 1996 and, in revised form, May 9, 1996.

1991 Mathematics Subject Classification. Primary 03D25, 03E60, 04A15; Secondary 03D30.

Key words and phrases. Post's problem, degree invariant operators, recursively enumerable degrees, computably enumerable degrees, Martin's conjecture, axiom of determinacy.

The first author's research was partially supported by the U.S. ARO through ACSyAM at the Mathematical Sciences Institute of Cornell University Contract DAAL03-91-C-0027, the IGC of Victoria University and the Marsden Fund for Basic Science under grant VIC-509.

The second author's research was partially supported by NSF Grant DMS-9503503 and the U.S. ARO through ACSyAM at the Mathematical Sciences Institute of Cornell University Contract DAAL03-91-C-0027.

(C) 1997 American Mathematical Society 
Lachlan [6] proved that if we require the degree invariance to be uniform in the sense that there is a function $h$ that takes the (pairs of) indices of reductions between $A$ and $B$ to (pairs of) indices of reductions between $W(A)$ and $W(B)$ then there is no such operator. In fact, Lachlan proved that if $\mathbf{d}$ is the degree of the function $h$ providing the uniformity then either $W(\mathbf{d})=\mathbf{d}^{\prime}$ or $W(W(\mathbf{d})) \leq W(\mathbf{d})$. In this way, Lachlan also characterized the jump operator as the only uniformly degree invariant computably enumerable operator which is always strictly greater than the identity on a cone (of degrees), i.e. on all $\mathbf{a} \geq_{T} \mathbf{c}$ for some degree $\mathbf{c}$.

In the setting of the Axiom of Determinacy (where, by Martin [7] there is a 0-1 valued countably complete measure defined on all the subsets of the Turing degrees for which being of measure 1 is equivalent to containing a cone), Martin made a sweeping conjecture (listed as Victoria Delfino Prize problem in [5], p. 279) that would entirely characterize the natural degree operators:

Conjecture 1. (Martin): Assume $Z F+A D+D C$. Then

I. If $f: 2^{\omega} \rightarrow 2^{\omega}$ is degree invariant then either $f$ is degree increasing on a cone or is constant (up to degree) on a cone.

II. The relation $\preceq$ on degree invariant functions defined by $f \preceq g$ iff $f(x) \leq_{T} g(x)$ on a cone is a prewellordering in which the immediate successor of any $f$ is its jump $f^{\prime}$ defined by $f^{\prime}(A)=f(A)^{\prime}$.

Of course, the assumption of determinacy is tied to the definability issue. If one assumes some sort of "definable" determinacy then one would hope to prove the conjecture for "definable" functions. In particular since determinacy is a theorem of ZFC for Borel $\left(\Delta_{1}^{1}\right)$ sets (Martin [8]), a proof of part II of the conjecture would almost certainly show that there is no degree invariant solution to Post's problem which is even a $\Delta_{1}^{1}$ operator let alone a computably enumerable $\left(\Sigma_{1}^{0}\right)$ one. (Consider the identity operator id. By II, its immediate successor is the Turing jump operator.) Thus Martin's Conjecture can be seen as asserting that the only natural operators on degrees are the jump operators and their iterates.

If one considers only uniformly degree invariant operators then Steel [13], Slaman and Steel [12] and Becker [1] have entirely settled the issue. The first proves II (and so generalizes Lachlan [6]), the second proves I and the third shows that every such operator greater than the identity is the jump operator associated with some pointclass in roughly the way the Turing jump is associated with the sets $\Sigma_{1}^{0}$ in $A$, the arithmetic jump ( $A$ goes to $A^{(w)}=\left\{\langle n, x\rangle \mid x \in A^{(n)}\right\}$ ) is associated with the sets arithmetic in $A$ and Kleene's hyperjump is associated with the sets $\Pi_{1}^{1}$ in $A$. On the other hand, almost nothing is known about degree invariant functions which are not already known to be uniform. The one exception is Slaman and Steel's remarkable result ([12]) that if $f$ is degree invariant and $f(\mathbf{x})<\mathbf{x}$ on a cone then $f$ is constant (up to degree) on a cone. At one end of the spectrum, Steel [13] has conjectured that all degree invariant functions are uniform and so a posteriori Martin's conjectures are true. On the other hand, Kechris [4] has conjectures that imply strong refutations of the conjectures, in particular, that there are degree invariant functions $f$ which are continuum many to one and so are very far from being jump operators (indeed they are not even above the identity on a cone).

In this note we considerably restrict the possible behavior of a degree invariant solution to Post's problem and give analogous information about more general definable degree invariant operators. In particular, we refute a number of appealing possibilities for solutions that are more than simply noncomputable and incomplete. 
One natural (if unlikely) such question asks if there is a computably enumerable half jump operator $H$. Such an operator would be a degree invariant computably enumerable operator $H$ such that $H(H(A)) \equiv_{T} A^{\prime}$ for every $A$. If we eliminate the crucial requirement of degree invariance, then Jockusch and Shore [3] (Corollary 3.4) prove that there indeed is such a computably enumerable operator (i.e. there is an $e$ such that, for every $\left.A, W_{e}^{W_{e}^{A}} \equiv_{T} A^{\prime}\right)$. Note that as shown in [3] any such half jump operator must always produce an intermediate computably enumerable degree (relative to its input), i.e. $A^{(n)}<_{T} H(A)^{(n)}<_{T} A^{(n+1)}$ for every $n$ (as $H^{2 n}(A)<_{T}$ $H^{2 n+1}(A)<_{T} H^{2 n+2}(A)$ while $H^{2 n}(A) \equiv_{T} A^{(n)}, H^{2 n+1}(A)=H^{2 n}(H(A)) \equiv_{T}$ $H(A)^{(n)}$ and $\left.H^{2 n+2}(A) \equiv_{T} A^{(n+1)}\right)$. Thus a weaker, but still strong, invariant solution to Post's problem would be a computably enumerable operator $W$ such that $W(A)$ is always of intermediate degree relative to $A$. We prove that there is no such operator. Indeed (assuming "definable determinacy"), any degree invariant definable solution to Post's problem must be low 2 or high ${ }_{2}$ on a cone. We begin with our main theorem for computably enumerable operators.

Theorem 2. If $W$ is a degree invariant computably enumerable operator which is at least the identity (i.e. $A \leq_{T} W(A)$ ) on a cone, then either $W(A)^{\prime \prime} \equiv_{T} A^{\prime \prime}$ on a cone or $W(A)^{\prime \prime} \equiv_{T} A^{\prime \prime \prime}$ on a cone.

Proof. The proof is essentially an analysis of how complicated is the desired uniformity information about $W$ and an application of Steel's result [13] on uniform operators. By our assumption that $A \leq_{T} W(A)$ on a cone, we may assume that $W$ is uniformly above $A$, i.e. of the form $W_{e}^{A} \oplus A$. (As we are only interested in the degree of $W(A)$ on a cone, we can simply replace $W$ by the operator giving $W(A) \oplus A$ which agrees with it up to degree on a cone and is of the desired form.) Let $\tilde{W}(A)$ contain the uniformity information for the degree invariance:

$$
\begin{gathered}
\tilde{W}(A)=\left\{\langle i, j, m, n\rangle \mid\{i\}^{A} \text { is total } \&\{j\}^{\{i\}^{A}}=A \&\{m\}^{W(A)}=W\left(\{i\}^{A}\right)\right. \\
\left.\&\{n\}^{\{m\}^{W(A)}}=W(A)\right\} .
\end{gathered}
$$

A straightforward Tarski-Kuratowski calculation (count the quantifiers) shows that $\tilde{W}(A) \leq_{T} W(A)^{\prime \prime}$ uniformly in $A$. (Note that we here use the assumption that $A$ is uniformly computable from $W(A)$ and that $W\left(\{i\}^{A}\right)$ is $\Sigma_{1}^{A}$.) On the other hand, a standard coding argument shows that $W(A)^{\prime \prime} \leq_{T} \tilde{W}(A)$ : Fix $i, j, n$ such that each is an index for the identity operator. Choose a computable function $g$ such that, for every $X,\{g(m)\}^{X}=X$ if $\{m\}^{X}$ is total and otherwise is not a total function. Finally, note that $\{m\}^{W(A)}$ is total if and only if $\langle i, j, g(m), n\rangle \in \tilde{W}(A)$. As the set of such $m$ is a complete $\Pi_{2}^{W(A)}$ set, we see that $W(A)^{\prime \prime} \leq_{T} \tilde{W}(A)$ as required. Thus $\tilde{W}(A) \equiv_{T} W(A)^{\prime \prime}$ (uniformly) and so $A^{\prime \prime} \leq_{T} \tilde{W}(A) \equiv_{T} W(A)^{\prime \prime} \leq_{T}$ $A^{\prime \prime \prime}$.

Now, if $\tilde{W} \equiv_{T} W^{\prime \prime}$ were uniformly degree invariant, Steel's results ([13], Lemma 2 ) would imply that $\tilde{W}(A) \equiv_{T} W(A)^{\prime \prime} \equiv_{T} A^{\prime \prime}$ on a cone or $\tilde{W}(A) \equiv_{T} W(A)^{\prime \prime} \equiv_{T}$ $A^{\prime \prime \prime}$ on a cone as required. We now show that $W^{\prime \prime}$ is uniformly degree invariant. As $\tilde{W} \leq W^{\prime \prime}$ we can fix indices $e, f$ such that, for any $i, j, A, B$ with $\{i\}^{A}=B$ and $\{j\}^{B}=A,\left\{\{e\}^{W(A)^{\prime \prime}}(i, j)\right\}^{W(A)}=W(B)$ and $\left\{\{f\}^{W(B)^{\prime \prime}}(i, j)\right\}^{W(B)}=W(A): e$ is the index of the procedure that, given $i$ and $j$, finds the least pair $\langle m, n\rangle$ such that $\langle i, j, m, n\rangle \in \tilde{W}(A)$ and then outputs $m ; f$ indexes the procedure which finds the same pair and then outputs $n$. Thus there are computable functions $h, k$ such 
that $W(B)^{\prime \prime}=\{h(i, j)\}^{W(A)^{\prime \prime}}$ and $W(A)^{\prime \prime}=\{k(i, j)\}^{W(B)^{\prime \prime}}$ as required to show uniform degree invariance. (Intuitively, to calculate the reduction from $W(A)^{\prime \prime}$ to $W(B)^{\prime \prime}$, for example, we first (using $\tilde{W}(A)=W(A)^{\prime \prime}$ calculate the index $m$ such that $\{m\}^{W(A)}=W(B)$. Then, using $W(A)^{\prime \prime}$, we calculate the index $\hat{m}$ such that $\{\hat{m}\}^{W(A)^{\prime \prime}}=\left(\{m\}^{W(A)}\right)^{\prime \prime}$.)

To generalize this theorem in the setting of determinacy assumptions, first note that all the calculations go through as before if $W$ is a $\Delta_{2}^{0}$ operator, i.e. there is a fixed $e$ such that $W(A)=\{e\}^{A^{\prime}}$ for every $A$. (The only point to notice is that $W\left(\{i\}^{A}\right)$ is, in this case, uniformly $\Delta_{2}^{A}$ and this fact suffices to show that $\{m\}^{W(A)}=W\left(\{i\}^{A}\right)$ is still $\Pi_{2}^{W(A)}$.) Indeed, as we are only interested in the behavior of the $W$ on a cone and $W$ is degree invariant, it suffices to have an $e$ such that $W(A)=\{e\}^{A^{\prime}}$ for every $A$ on some perfect pointed tree. (A set $T$ of finite binary sequences $\sigma$ is a tree (on 2) if it is closed under initial segments. A tree $T$ is perfect if for every $\sigma \in T$ there are incompatible $\tau$ and $\tau^{\prime}$ extending $\sigma$, i.e. $\sigma \subseteq \tau, \tau^{\prime}$ but neither of $\tau, \tau^{\prime}$ extends the other. The tree is pointed if it is computable in every $x \in[T]$ where we say that $x$ is on $T$, written $x \in[T]$, if $x \in 2^{\omega}$ and $\forall n \exists \sigma \in T(x \uparrow n \subseteq \sigma)$. Thus the sets on a pointed perfect tree $T$ consist of representatives of the degrees in the cone above the degree of $T$.) The next ingredient in our analysis is that, under the assumption of "definable" determinacy, for any definable degree invariant operator $W$ such that $A<_{T} W(A)<_{T} A^{\prime}$ for all $A$ on a perfect pointed tree $T$ there is a fixed $e$ and a perfect pointed subtree $\hat{T}$ of $T$ such that $W(A)=\{e\}^{A^{\prime}}$ for all $A$ on $\hat{T}$. This follows from a classic result of Martin [7]. Thus we can prove our main theorem for any definable degree invariant operator.

Theorem 3. If $W$ is a definable degree invariant operator such that $A \leq_{T} W(A)$ $\leq_{T} A^{\prime}$ for all $A$ on some perfect pointed tree $T$ then, under the assumption of definable determinacy, there is a perfect pointed subtree $\hat{T}$ of $T$ such that either $W(A)^{\prime \prime} \equiv_{T} A^{\prime \prime}$ for all $A$ on $\hat{T}$ or $W(A)^{\prime \prime} \equiv_{T} A^{\prime \prime \prime}$ for all $A$ on $\hat{T}$. In particular if $W$ is $\Delta_{1}^{1}$ then, provably in $Z F C$, there is such a tree $\hat{T}$.

Proof. The proof is as before once we refine $T$ to a subtree on which $W(A)=\{e\}^{A^{\prime}}$ as described above. If $W$ is $\Delta_{1}^{1}$ then the only determinacy needed is for Borel sets and so is provable in ZFC by Martin [8].

One consequence of this formulation of our result is a restriction on the possibility of naturally defining a single degree in the structure $\mathcal{R}$ of (computably) enumerable degrees. If, by a natural definition, we mean one that relativizes to every degree then any natural definition of a single nontrivial degree in $\mathcal{R}$ defines an arithmetic degree invariant operator $W$ such that $A<_{T} W(A)<_{T} A^{\prime}$ for every $A$. By the last

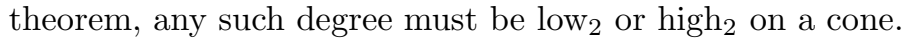

\section{REFERENCES}

1. Becker, H., A. characterization of jump operators, Journal of Symbolic Logic 53 (1988), 708728. MR 90a:03067

2. Friedberg, R. M., Two recursively enumerable sets of incomparable degrees of unsolvability, Proc. Nat. Ac. Sci. 43 (1957), 236-238. MR 18:867a

3. Jockusch, C. G. Jr. and Shore, R. A., Pseudo-jump operators I: the r.e. case, Trans. Am. Math. Soc. 275 (1983), 599-609. MR 84c:03081 
4. Kechris, A. S., The structure of Borel equivalence relations in Polish spaces, in Set Theory and the Continuum, H. Judah, W. Just and W. H. Woodin eds., MSRI Publications 26, Springer-Verlag, Berlin, 1992. MR 94h:03093

5. Kechris, A. S. and Y. Moschovakis, eds., Cabal Seminar 76-77, LNMS 689, Springer-Verlag, Berlin, 1978. MR 80b:03004

6. Lachlan, A. H., Uniform enumeration operators, Journal of Symbolic Logic 40 (1975), 401409. MR 52:62

7. Martin, D. A., The axiom of determinacy and reduction principles in the analytic hierarchy, Bull. Am. Math. Soc. 74 (1968), 687-689. MR 37:2607

8. Martin, D. A., Borel determinacy, Ann. Math. (2) 102 (1975), 363-371. MR 53:7785

9. Muchnik, A. A., On the unsolvability of the problem of reducibility in the theory of algorithms, Dokl. Akad. Nauk SSSR N. S. 108 (1956), 29-32.

10. Post, E. L., Recursively enumerable sets of positive integers and their decision problems, Bull. Am. Math. Soc. 50 (1944), 84-316 MR 6:29f

11. Sacks, G. E., Degrees of unsolvability, Annals of Math. Studies 55, Princeton University Press, Princeton NJ 1963; $2^{\text {nd }}$ ed. 1966. MR 32:4013

12. Slaman, T. and J. R. Steel, Definable functions on degrees, in Cabal Seminar 81-85, A. S. Kechris, D. A. Martin and J.. R. Steel eds., LNMS 1333 (1988) Springer-Verlag, Berlin, 37-55. MR 89m:03033

13. Steel, J. R., A classification of jump operators, Journal of Symbolic Logic 47 (1982), 347-358. MR 84i:03085

Department of Mathematics, Victoria University of Wellington, P. O. Box 600, Wellington, New Zealand

E-mail address: rod.downey@vuw.ac.nz

Department of Mathematics, Cornell University, Ithaca, New York 14853

E-mail address: shore@math.cornell.edu 